\title{
Physical Activity Counseling for Children With Cystic Fibrosis
}

\author{
Fiona J Moola PhD, Eric Garcia MSc, Elizabeth Huynh MA, Lauren Henry MOT, \\ Shannon Penfound MA, Raquel Consunji-Araneta MD, and Guy EJ Faulkner PhD
}

\begin{abstract}
BACKGROUND: Physical activity is recommended as a component of the cystic fibrosis (CF) treatment regimen. However, to date, there is limited research examining the effects of behavioral counseling interventions aimed at increasing physical activity. The aim of this study was to assess the feasibility of a theoretically informed, parent-mediated counseling intervention in increasing habitual physical activity and quality of life among children and youth with CF. METHODS: Participants were recruited from the pediatric respirology clinic at the Children's Hospital of Winnipeg. Participants ranged in age between 8 and $18 \mathrm{y}$. A randomized control feasibility trial was implemented, and participants were randomized to the intervention $(n=7)$ or control group $(n=6)$. Intervention group participants engaged in 4 counseling sessions to examine the acceptability and feasibility of physical activity counseling in the care of children with $\mathrm{CF}$. The primary outcome was study feasibility, as measured by recruitment, retention, adherence, acceptability, and the frequency of adverse events. Secondary outcomes included physical activity and quality of life, as measured by accelerometry and the Pediatric Quality of Life Inventory. RESULTS: Thirteen subjects completed the study. No adverse events were found in this trial. The intervention was found to be feasible and acceptable with good recruitment, retention, adherence, and acceptability. Positive trends were also reported in terms of increases in physical activity, reductions in time spent being sedentary, and improvements in most dimensions of quality of life pre- to post-intervention. CONCLUSIONS: The findings suggest that counseling is feasible for the CF community. An appropriately powered randomized controlled trial is required in the future to investigate the utility of counseling as a means to enhance quality of life and physical activity behavior. Key words: cystic fibrosis; counseling; physical activity; quality of life; randomized control trial; feasibility. [Respir Care 2017;62(11):1466-1473. (c) 2017 Daedalus Enterprises]
\end{abstract}

\section{Introduction}

Physical activity and exercise may improve physical and psychosocial health as well as quality of life in people with cystic fibrosis (CF). ${ }^{1}$ In addition to enhancing per-

\footnotetext{
Dr Moola is affiliated with Holland Bloorview Kids Rehabilitation Hospital, East York, Canada and the Dalla Lana School of Public Health at the University of Toronto, Toronto, Canada. Mr Garcia is affiliated with Kinesiology and Recreation Management at the University of Manitoba, Manitoba, Canada. Ms Huynh is affiliated with CancerCare Manitoba, Manitoba, Canada. Ms Henry is affiliated with the University of Manitoba, Manitoba, Canada. Ms Penfound is affiliated with the Wellness Institute, Winnipeg, Canada. Dr Consunji-Araneta is a affiliated with the Children's Hospital of Winnipeg, Winnipeg, Canada and the University of Manitoba, Department of Pediatrics and Child Health, Section of Respirology, Manitoba, Canada. Dr Faulkner affiliated with the School of Kinesiology at the University of British Columbia, Vancouver, Canada.
}

ceptions of wellness and quality of life, numerous studies have found that exercise training is associated with notable physical benefits for people with CF. There have been 11 randomized controlled trials in the field of physical activity and CF. ${ }^{2}$ Researchers have reported short-term improvements in physical and psychosocial outcomes as a result of exercise training in young $\mathrm{CF}$ populations. ${ }^{3-7} \mathrm{Re}-$ search teams have reported improvements in respiratory

\footnotetext{
This study was supported by Research Manitoba. The authors have disclosed no conflicts of interest.

Correspondence: Fiona J Moola PhD, Bloorview Research Institute, Holland Bloorview Kids, Rehabilitation Hospital, 150 Kilgour Road, East York, ON M4G 1R8, Canada. E-mail: fiona.moola@utoronto.ca.
}

DOI: $10.4187 /$ respcare.05009 


\section{Physical Activity Counseling IN CF}

function $^{8,9}$ as well as quality of life for people with $\mathrm{CF}$ randomized to exercise-intervention groups. Researchers are beginning to examine the impact of exercise on other health parameters in people with $\mathrm{CF}$, such as postural tone. ${ }^{10}$ Researchers are also investigating the impact of unconventional forms of physical activity on the health in people with $\mathrm{CF}$, such as the Chinese martial art form tai chi. ${ }^{11}$ In all cases, engagement in physical activity was associated with either improvements in health status or no change. In no cases were physical activity and exercise associated with adverse outcomes. Importantly, the association between aerobic fitness and survival is perhaps the most compelling rationale for the incorporation of physical activity promotion within the care of people with $\mathrm{CF}$. Higher physical activity levels and peak $\dot{\mathrm{V}}_{\mathrm{O}_{2}}$ are positively related to survival in CF. ${ }^{12,13}$ Physical activity, and exercise in particular, may slow the rate of lung function decline over time and contribute toward survival. ${ }^{3}$

However, most youth with $\mathrm{CF}$ do not engage in the recommended amounts of physical activity. Physical activity among CF youth tends to plummet further during adolescence. ${ }^{14}$ Although the reasons for physical inactivity in the CF population are not entirely known, it is thought that young people with CF face numerous barriers to physical activity participation, such as low self-efficacy and competing time demands. ${ }^{15}$

Although research in the field of physical activity and $\mathrm{CF}$ is developing, there are several gaps in the existing literature that necessitate novel approaches to enhancing physical activity. Existing interventionists tend to adopt a conventional exercise training approach. ${ }^{16}$ Such programs may lack sensitivity to participants' local environments and interests. They are cost- and resource-intensive, and, upon termination of the program, these researchers invariably encounter difficulties with sustainability.3,17,18 A complementary yet arguably more realistic approach is to encourage increases in habitual physical activity by integrating opportunities to be active into patients' daily routines in the context of their own lives at home. In addition, although theory-based interventions are more effective in increasing physical activity than non-theoretical approaches, thus far, researchers have not created interventions that are guided by theory. ${ }^{19,20}$ Before developing broader-scale, fully powered interventions, ${ }^{21}$ it is imperative to discern information about feasibility and to assess the acceptability of interventions for people with CF in the context of their own lives.

By addressing these limitations, the aim of this study was to assess the feasibility of a theoretically informed, family-mediated counseling intervention in increasing habitual physical activity and quality of life among children and youth with CF. Given the feasibility nature of the trial, our outcomes were focused on the feasibility of enrolling, randomizing, and assessing physical activity using acceler-

\section{QUICK LOOK}

\section{Current knowledge}

People living with cystic fibrosis are at an increased risk of psychosocial morbidity, such as depression, anxiety, and poor quality of life. Regular physical activity can help to improve psychosocial well-being. Some researchers suggest that physical activity also slows the rate of lung function decline in patients with cystic fibrosis.

\section{What this paper contributes to our knowledge}

Subjects demonstrated increased physical activity behavior and quality of life. Participants also felt that the study was feasible. A larger, fully powered national trial is needed to determine whether counseling is an effective means to increase physical activity and quality of life in this population.

ometry, administering measurements of quality of life, and identifying rates of recruitment and retention of participants.

\section{Methods}

Ethical approval was obtained from the Education and Nursing Research Ethics Board and the University of Manitoba. Parental consent was obtained for all participants.

A randomized controlled trial of feasibility was conducted. Subjects were randomized to a physical activity program or to a standard care group. Because this was a randomized controlled trial of feasibility only, there was no calculation of sample size.

\section{Subjects}

In March 2014, the research coordinator contacted eligible subjects in the children's respiratory medicine clinic via telephone at the Children's Hospital of Winnipeg to inquire about participating in the study. Children between the ages of 8 and $18 \mathrm{y}$ with a positive diagnosis of $\mathrm{CF}$ were considered for this study. During the study, we were inclusive of sex, culture, race, and socioeconomic status. Transplant candidates were not considered for this study. Medically unstable children, such as those with acute respiratory distress or infection, were not contacted for participation. Patients with cognitive and intellectual disabilities were excluded from the study. This is due to the fact that $\mathrm{CF}$ Chatters program participants require sufficient comprehension and communication abilities. Due to the counseling-based nature of the study, participants were required to travel to the hospital every 2 weeks. Therefore, 


\section{Physical Activity Counseling IN CF}

Table 1. Participants' Characteristics

\begin{tabular}{|c|c|c|c|c|c|c|c|c|}
\hline Participant & Age $(y)^{*}$ & Sex & $\begin{array}{l}\text { Weight } \\
(\mathrm{kg})\end{array}$ & $\begin{array}{l}\text { Height } \\
(\mathrm{cm})\end{array}$ & $\begin{array}{c}\text { BMI } \\
\text { Percentile }\end{array}$ & $\begin{array}{c}\text { Predicted } \\
\text { Normal FEV }_{1}(\mathrm{~L})\end{array}$ & $\begin{array}{c}\text { Measured } \\
\text { FEV }_{1} \text { Value (L) }\end{array}$ & $\begin{array}{c}\text { Percent Predicted } \\
\text { Normal FEV }_{1} \dagger\end{array}$ \\
\hline \multicolumn{9}{|l|}{ Intervention } \\
\hline 1 & 8 & Female & 22.5 & 129 & 3 & 1.54 & 1.55 & 100 \\
\hline 2 & 8 & Female & 23 & 129 & 6 & 1.54 & 1.73 & 112 \\
\hline 3 & 16 & Male & 61 & 185 & 10 & 4.68 & 3.95 & 84 \\
\hline 4 & 10 & Male & 38.6 & 143.7 & 78 & NA & NA & NA \\
\hline 5 & 18 & Female & 63.8 & 167 & 78 & 3.3 & 3.86 & 116 \\
\hline 6 & 12 & Female & 34 & 143 & 22 & 2 & 2.09 & 104 \\
\hline 7 & 8 & Female & 28.8 & 130 & 66 & 1.57 & 1.67 & 106 \\
\hline \multicolumn{9}{|l|}{ Control } \\
\hline 1 & 9 & Female & 27.9 & 137 & 18 & 1.78 & 1.21 & 68 \\
\hline 2 & 12 & Female & 36.6 & 143 & 46 & 2.02 & 2.01 & 99 \\
\hline 3 & 15 & Male & 61.5 & 174 & 46 & 3.89 & 2.97 & 76 \\
\hline 4 & 12 & Male & 38.8 & 157 & 13 & 2.64 & 2.56 & 96 \\
\hline 5 & 13 & Male & 62.1 & 165 & 86 & 3.1 & 2.99 & 96 \\
\hline 6 & 10 & Female & 26.1 & 130 & 19 & 1.58 & 1.4 & 88 \\
\hline \multicolumn{9}{|c|}{$\begin{array}{l}\text { * Mean age }=11.6 \text { y old; median age }=12 \text { y old. } \\
\dagger \text { Mean percent predicted }=95.4 \% \text {; median percent predicted }=97.5 \% \text {. } \\
\text { BMI = body mass index } \\
\mathrm{NA}=\text { not applicable; unable to reliably perform consistent spirometry } \mathrm{m}\end{array}$} \\
\hline
\end{tabular}

patients residing outside of Winnipeg in the surrounding regions were excluded.

\section{Randomization}

Using opaque sealed envelopes, the participants were randomized by an independent investigator to the intervention or control group. Participants randomized to the standard care (control group) continued with regular follow-up care in the CF clinic at the Children's Hospital of Winnipeg. There was no researcher contact with control group participants during this 8-week period.

\section{Intervention}

Intervention development included qualitative research with parents and children. This allowed us to develop a grounded theory of physical activity in CF for the purposes of guiding intervention development. Readers should refer to Moola et al ${ }^{15}$ for a further description of our grounded theory of physical activity in CF. Briefly, we developed an 8-week family-mediated physical activity counseling program termed CF Chatters. We define counseling in this context as a person- and family-centered consultation approach to increasing physical activity. ${ }^{22}$ Guided by the CF Chatters manual, the participants collaboratively worked on physical activity content with the counselors in a context of empathy and support. In all cases, a parent attended the session to support subjects' physical activity goals. The content of the intervention was based upon and informed by our foundational qualitative work, which identified disease-specific barriers and facilitators to physical activity. The aim of the manual is to encourage participants to focus on developing self-regulatory skills for the self-management of physical activity. Utilizing a range of behavior change techniques, we aimed to enhance participants' physical activity self-efficacy, ability to overcome physical activity barriers, and confidence to resume activity after prolonged periods of illness and hospitalization. Sessions included discussion prompts and written exercises on topics such as learning how to selfmonitor daily activity, setting goals, planning for setbacks, and overcoming barriers to physical activity. Baseline information was obtained from all participants before commencing the study (Table 1). Baseline information included age, sex, weight, height, body mass index percentile, and lung function. Baseline measurements of physical activity and quality of life were obtained. Anthropometry and spirometry were performed. For anthropometry, height and weight measurements were taken.

Height and participant sex were used to obtain the predicted normal values for spirometry. The reference equation used was from Wang et al. ${ }^{23}$ Forced expiratory maneuvers were performed using acceptability standards as set by the American Thoracic Society. After selecting 3 reproducible maneuvers, the best $\mathrm{FVC}$ and $\mathrm{FEV}_{1}$ values were recorded.

The participants met with physical activity counselors every 2 weeks over the course of 8 weeks. Each session was approximately $90 \mathrm{~min}$ in duration. The current $\mathrm{CF}$ 


\section{Physical Activity Counseling IN CF}

Chatters manual has been developed to approximate the developmental abilities of 13-y-old children. Although we did not develop formal versions of the manual based on age, the counselors adapted the content to the participants' developmental age by altering the language used to explain workbook content. Thus, attempts were made to deliver the content of the manual in an age-appropriate fashion. The physical activity counselors delivered the manualized content to participants, discussed physical activity goals and behaviors, taught physical activity selfmanagement skills, and provided empathy and support for physical activity challenges. We frequently reminded participants about their upcoming CF Chatters sessions using text, telephone, and email. The CF Chatters physical activity counselors (fourth year undergraduate level) were trained for $6 \mathrm{~h}$ each on how to deliver the activity sessions to participants. Please contact the first author for a copy of the CF Chatters manual. Measurements were repeated again at the end of the 12-week period.

\section{Primary and Secondary Outcome Measurements}

Feasibility. The primary outcome measure for this study was the feasibility of the CF Chatters program. We measured feasibility according to (1) recruitment, (2) retention, (3) adherence, (4) acceptability, and (5) the frequency of any adverse events during the intervention. Recruitment was measured by assessing the percentage of subjects who consented to the study after being contacted by the researcher. Retention was measured by calculating the percentage of enrolled participants who completed the study as well as the percentage of enrolled participants who dropped out. Adherence was measured by recording the percentage of study instruments, assessments, and counseling sessions that the participants completed. Acceptability was assessed during a qualitative exit interview by asking the participants to address issues of convenience, feasibility, and ease. Although all subjects were asked about acceptability, including parents, for the purposes of this paper, we report on the findings from child participants only. The frequency of adverse events was assessed through monitoring the daily activities that transpired in the study and recording any negative events that occurred during the intervention. Feasibility outcomes were collected during the entire course of the study, including attendance records kept by the counselors.

Physical Activity. Secondary outcomes included changes in minutes engaged in moderate and vigorous physical activity and changes in sedentary time (min). Moderateto-vigorous physical activity is any activity the participant performed that would accumulate $>2,296$ counts $/ \mathrm{min}$. Physical activity and sedentary time were assessed through the use of accelerometry. Standard protocols were followed for screening and analyzing accelerometer data. To have a valid accelerometer file, the participant had to wear the accelerometer for at least $4 \mathrm{~d}$. This included at least 3 weekdays and 1 weekend day. ${ }^{24,25}$ The 4 -d wear time protocol has been reliably used in other $\mathrm{CF}$ accelerometer trials. A valid day of accelerometer data was defined as $\geq 8 \mathrm{~h}$ of wear time. ${ }^{26,27}$ The participants started to wear the accelerometer as soon as they received them. They were instructed to put the accelerometer on when waking up and to wear it all day every day for a period of 1 week. They were instructed to only remove the device when showering and going to bed. A 5-s interval (epoch) was used to capture rapid transitions in activity typical in children. ${ }^{28,29}$ We used the cutoff points that have been developed and employed with a pediatric CF population previously. ${ }^{25,30}$ In doing so, moderate and vigorous physical activity was defined as $>2,296$ accelerometer counts/min. Sedentary time was defined as $<100$ counts/min.

Quality of Life. The Pediatric Quality of Life Inventory was administered at baseline and week 12. The Pediatric Quality of Life Inventory is a well-validated psychometric tool used to measure quality of life in chronically ill children. ${ }^{31}$ Although it was originally developed for children with cancer, it has been adapted for wide use across pediatric chronic disease groups. As a generic quality-of-life tool, it allows for broad comparisons in quality of life among different pediatric disease groups. The tool assesses quality of life in 4 domains, including physical, emotional, school-related, and social health. The tool was completed in writing by each participant pre- and post-intervention.

\section{Analysis}

Descriptive statistics (\%) are reported for the feasibility outcomes. The statistical method employed was a descriptive statistics analysis, including before and after comparison of means and medians between intervention and control groups. For the secondary outcomes, data management and statistical analysis were performed using Statistica (StatSoft, Tulsa, Oklahoma). Others have noted that feasibility studies are not intended for hypothesis testing. ${ }^{32,33}$ Given that they are not powered to detect between-group differences, feasibility studies should not be used to determine sample size calculations for an efficacy study. ${ }^{33}$ Feasibility should be tested to ensure that primary outcome assessment can be conducted and to explore the sensitivity of these primary outcomes to change. ${ }^{34}$ Means, medians, and SD values are thus presented as results. For participants who were missing one data point, we carried the last data point forward to replace the missing data point. This occurred in the case of 3 participants who failed to complete quality-of-life post-tests (Fig. 1). This 


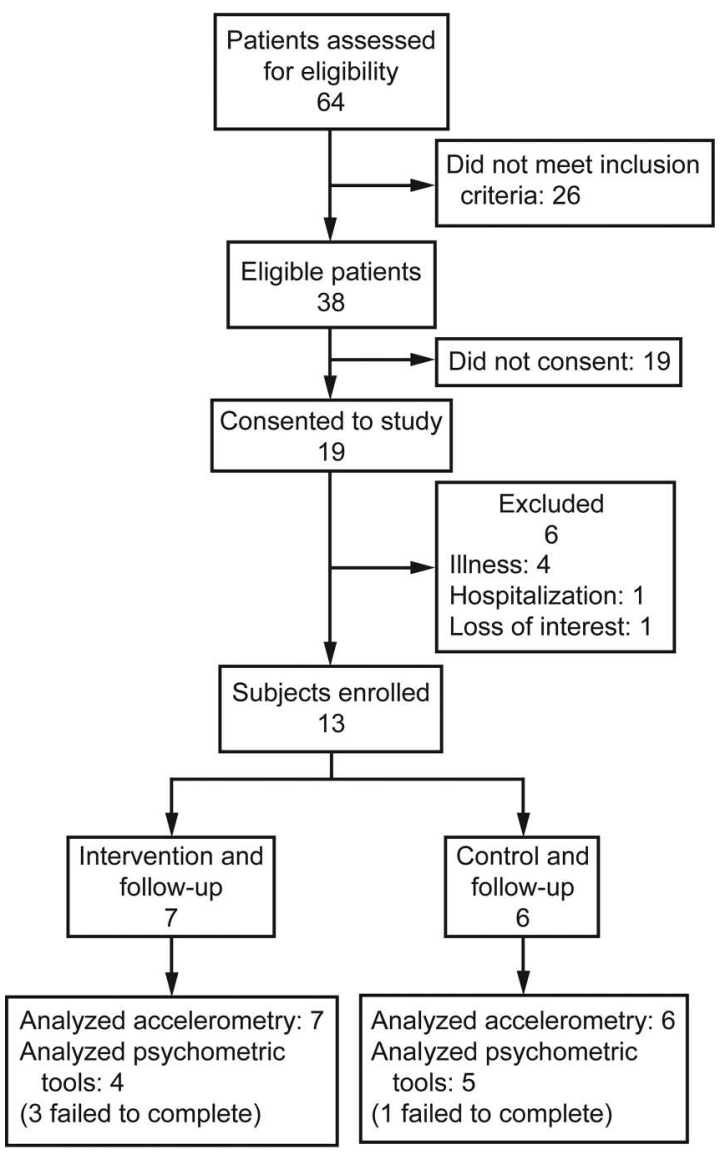

Fig. 1. Flow chart.

last-observation-carried-forward method is a very common approach for handling missing data, although it is not without potential for bias. ${ }^{35}$

\section{Results}

\section{Feasibility}

After all exclusion criteria were employed, 38 of 64 subjects were identified as eligible to participate. Of the 38 eligible participants who were contacted in the pediatric CF clinic, 19 consented to participation. This represents a recruitment rate of $50 \%$. Six participants dropped out of the study before baseline measurements due to illness $(n=4)$, hospitalization $(n=1)$, or loss of interest $(n=1)$. All 13 participants completed physical activity measurements pre- and post-intervention. Twelve participants completed the quality-of-life measurement at baseline. Nine participants completed them post-intervention. All intervention arm participants $(n=7)$ attended and completed the entire counseling course. These counseling sessions were held at the hospital.

\section{Sample}

Sample characteristics are presented in Table 1. There were 13 participants in the study, including 7 in the intervention group and 6 in the control group. In the intervention group, age ranged between 8 and $18 \mathrm{y}$. In the control group, age ranged between 9 and $15 \mathrm{y}$. There were 5 girls and 2 boys in the intervention group. There were 3 girls and 3 boys in the control group. For girls in the intervention group, height ranged between 129 and $167 \mathrm{~cm}$. For boys in the intervention group, height ranged between 143.7 and $185 \mathrm{~cm}$. Height for girls in the control group ranged from 130 to $143 \mathrm{~cm}$. Height for boys in the control group ranged from 157 to $174 \mathrm{~cm}$. BMI percentile is the standard measurement for weight in pediatrics. BMI percentile for girls in the intervention group ranged from 3 to 78 . BMI percentile for boys in the intervention group ranged from 10 to 78 . BMI percentile for girls in the control group ranged from 18 to 46 . BMI percentile for boys in the control group ranged from 13 to 86 . In our clinic, we use spirometry as a measure of disease severity. Spirometry values are reported as a percentage of the predicted normal value. The $\mathrm{FEV}_{1}$, expressed as a percentage of predicted normal, ranged between 84 and $116 \%$ among intervention group participants and between 68 and $99 \%$ for control group participants. The median measured $\mathrm{FEV}_{1}$ in the intervention group was $105 \%$, and the median $\mathrm{FEV}_{1}$ in the control group was $92 \%$. Intervention group participant 4) was not included in this calculation. This participant was not able to reliably perform consistent spirometry maneuvers.

All participants reported that the intervention was convenient, easy, and acceptable. The participants felt that the program was distinctly different from routine medical appointments. They enjoyed making relationships with familiar faces and developing a sense of rapport with the counselor. They felt that the program enhanced accountability and loyalty to the counselor. They stated that the counseling program was easy and convenient, providing a safe space to discuss health and activity. Details of the process-based elements of the study are reported in a previously published paper. ${ }^{21}$

\section{Secondary Outcomes}

Physical Activity. Complete accelerometry data were collected for all 13 participants. Minutes of moderate and vigorous physical activity and sedentary time at baseline and after week 12 are presented in Figures 2 and 3 for both groups, respectively. At baseline, the control group engaged in more moderate and vigorous physical activity (control: $48 \pm 14 \mathrm{~min} / \mathrm{d}$; median: $54 \mathrm{~min} / \mathrm{d}$; intervention: $43 \pm 5 \mathrm{~min} / \mathrm{d}$; median: $42.5 \mathrm{~min} / \mathrm{d}$ ) and sedentary time (control: $604 \pm 105 \mathrm{~min} / \mathrm{d}$; median: $561 \mathrm{~min} / \mathrm{d}$; interven- 
tion: $540 \pm 80 \mathrm{~min} / \mathrm{d}$; median: $506.5 \mathrm{~min} / \mathrm{d}$ ) than the intervention group. After 12 weeks, the intervention group participants demonstrated an increase in moderate and vigorous physical activity of $6 \mathrm{~min} / \mathrm{d}$ (to $49 \pm 21 \mathrm{~min} / \mathrm{d}$; median: $49.5 \mathrm{~min} / \mathrm{d}$ ), whereas the control group participants stayed approximately the same $(47 \pm 15 \mathrm{~min} / \mathrm{d}$; median: $42 \mathrm{~min} / \mathrm{d}$ ). The intervention group participants also demonstrated a larger decrease in sedentary time of $49 \mathrm{~min} / \mathrm{d}(491 \pm 54 \mathrm{~min} / \mathrm{d}$, median: $506.5 \mathrm{~min} / \mathrm{d})$ as com-

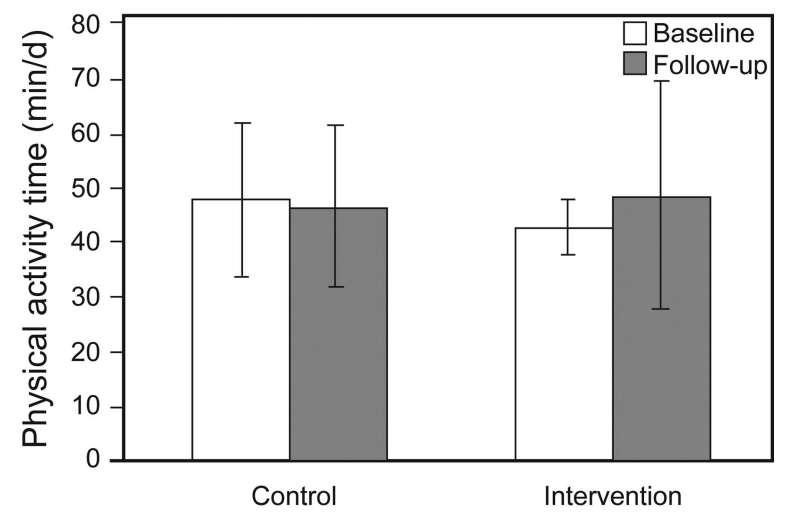

Fig. 2. Comparison of moderate-to-vigorous physical activity expressed as mean \pm SD in $\mathrm{min} / \mathrm{d}$ between the control group $(n=7)$ and the intervention group $(n=6)$ at baseline and 12-week followup.

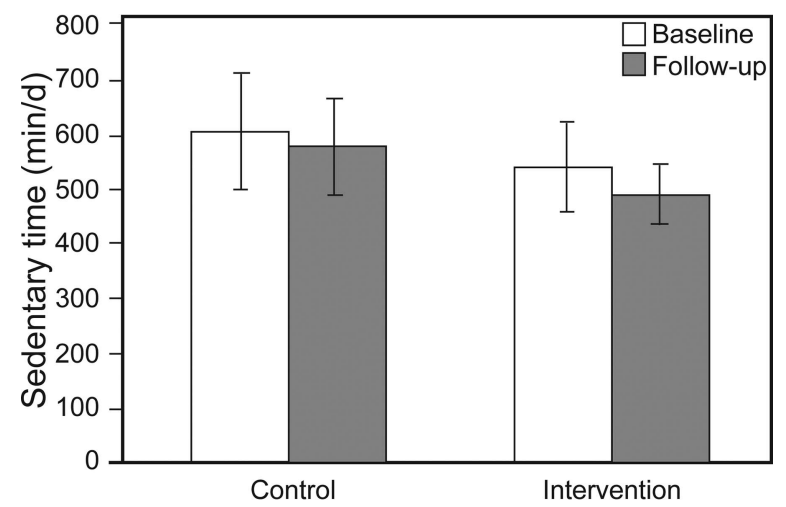

Fig. 3. Comparison of sedentary time expressed as mean $\pm S D$ in $\mathrm{min} / \mathrm{d}$ between the control group $(n=7)$ and intervention group $(n=6)$ at baseline and 12-week follow-up. pared with the $27-\mathrm{min} / \mathrm{d}$ decrease in the control group (577 $\pm 87 \mathrm{~min} / \mathrm{d}$; median: $561 \mathrm{~min} / \mathrm{d}$ ) (see Figs. 2 and 3).

Quality of Life. Twelve of 13 participants completed the psychometric tools at baseline. At follow-up, 9 of 12 participants completed the psychometric tool assessment. For the intervention group, there was a noticeable increase in the physical well-being dimension in comparison with the other dimensions (see Table 2). More modest improvements in emotional and school dimensions were observed as well as a decline in social well-being. In contrast, there were declines in physical, emotional, and social dimensions among control participants.

\section{Discussion}

In this study, we assessed the feasibility of a parentmediated physical activity-counseling program for pediatric subjects with $\mathrm{CF}$ at the Children's Hospital of Winnipeg. We also assessed the impact of the program on secondary outcomes, such as physical activity behavior and quality of life. Given promising feasibility and secondary outcomes, we can discern from these preliminary findings that the CF Chatters intervention is feasible for young people with $\mathrm{CF}$.

Our recruitment rate was $50 \%$, and our retention rate was $86 \%$. A few research teams failed to report recruitment rates ${ }^{6,7}$ in their randomized controlled trials. However, one study reported a recruitment rate of $44 \%$ and a retention rate of $90 \% .^{3}$ Other studies have reported retention rates of $82 \%,{ }^{5} 86 \%, 4,7$ and $90 \% .{ }^{6}$ Additionally, there have been reports including recruitment rates of $34 \%^{5}$ and $46 \% .{ }^{4}$ With the exception of one study, our recruitment and retention rates appear to be consistent with what has been reported in the previous literature. ${ }^{3}$ The majority of the subjects who dropped out of our study did so due to illness, hospitalization, or lack of interest (80\%).

Adherence to our counseling sessions was $100 \%$. Other studies have noted better adherence to exercise and physiotherapy in the context of research than clinical practice. ${ }^{3}$ Adherence was not reported in some studies. ${ }^{6,7}$ Although others did not keep explicit measurements of adherence,

Table 2. Pediatric Quality of Life Inventory Dimension Scores

\begin{tabular}{|c|c|c|c|c|}
\hline \multirow{2}{*}{ Domains } & \multicolumn{2}{|c|}{ Control $(n=6)$} & \multicolumn{2}{|c|}{ Intervention $(n=6)$} \\
\hline & Pre & Post & Pre & Post \\
\hline Physical & $91.15 \pm 8.25 ; 92.19$ & $82.81 \pm 18.41 ; 85.94$ & $73.44 \pm 12.30 ; 70.31$ & $78.13 \pm 15.81 ; 73.44$ \\
\hline Emotional & $80 \pm 14.14 ; 80$ & $73.33 \pm 22.51 ; 77.5$ & $66.67 \pm 21.37 ; 67.5$ & $67.5 \pm 19.17 ; 72.5$ \\
\hline Social & $98.96 \pm 2.55 ; 100$ & $96.67 \pm 5.16 ; 100$ & $80 \pm 20.74 ; 82.5$ & $73.33 \pm 17.80 ; 70$ \\
\hline School & $61.25 \pm 24.17 ; 61.25$ & $64.17 \pm 26.72 ; 65$ & $67.5 \pm 16.95 ; 62.5$ & $68.33 \pm 9.83 ; 67.5$ \\
\hline
\end{tabular}

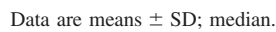




\section{Physical Activity Counseling IN CF}

they have suggested adherence difficulties. ${ }^{4}$ Our $100 \%$ adherence is encouraging and suggests that once participants have committed themselves to the counseling sessions, they are likely to adhere. This also suggests that participants might feel loyal and accountable to counselors in the program, with these relationships serving to better facilitate adherence. The high adherence rate is encouraging, given the general difficulty with adherence that this population reports. Based on a comparison with the general literature, our trial appears to be acceptable and feasible for young people with CF. This is also supported by participant reports that they enjoyed participating in the intervention.

Promising and positive trends were found for secondary outcomes in this study, including physical activity and quality of life, that need to be further investigated in the context of a larger, fully powered randomized controlled trial. A 6-min/d increase in moderate and vigorous physical activity was observed in the intervention group, from baseline to week 12. No increase in moderate and vigorous physical activity was observed in the control group from baseline to week 12. CF Chatters intervention group participants were engaging in approximately $42 \mathrm{~min}$ more moderate and vigorous physical activity per week, relative to the control group. Intervention group participants reduced their sedentary time by $49 \mathrm{~min} / \mathrm{d}$. A 27 -min reduction in sedentary time per day was observed in the control group.

There is growing interest in the health benefits of reducing sedentary time among youth and adults. ${ }^{36}$ Since the intervention group participants demonstrated a reduction in sedentary time and an increase in moderate and vigorous physical activity, the CF Chatters program might be an effective means to promote activity and reduce sedentariness among young people with CF. Given the association between increased activity and enhanced survival in $\mathrm{CF},{ }^{3}$ this promising trend toward enhanced activity requires further investigation in a larger trial.

Similarly, participants' scores on physical well-being, emotional well-being, and school functioning increased in the intervention group. An enhanced sense of physical well-being might be related to the greater physical activity that the children were engaging in during the intervention. It is well documented that enhanced physical activity behavior can improve perceptions of physical functioning in children with chronic illnesses. ${ }^{37}$ Because children with $\mathrm{CF}$ report physical malaise, such as difficulty breathing and fatigue, interventions that enhance perceptions of physical functioning are important for this population. ${ }^{38}$ Given the preponderance of psychosocial morbidity in young people with $\mathrm{CF}$, such as depression and anxiety, ${ }^{39}$ interventions such as CF Chatters that enhance emotional well-being are important. We did, however, encounter some difficulty in obtaining all of the post-test quality- of-life measurements. Strategies, such as the use of incentives to increase completion of psychometric tools, could be employed in the future.

The strengths associated with this feasibility study include the randomized design, the implementation of a theoretically informed intervention, and the use of objective physical activity measurements. Given the association between physical activity levels and improved life expectancy in CF, it is critical to accurately assess activity levels using objective measurements, such as accelerometry. However, there were several limitations associated with the study. Participants and researchers were not blinded to treatment allocation, which introduces a level of bias in the study. Mediators of behavior change (eg, self-efficacy) were not assessed. In our efforts to be flexible and allow participants to schedule convenient sessions, we were not always able to meet exactly every 2 weeks, which might have compromised the study protocol. There is also a need to create developmentally appropriate versions of the $\mathrm{CF}$ Chatters manual. Additionally, we chose to implement the generic pediatric quality-of-life tool because it is an easyto-use, well-validated, and robust inventory to employ with various pediatric disease groups. Our participants found the disease-specific Cystic Fibrosis Questionnaire-Revised tool to be much more cumbersome and confusing. Nevertheless, our failure to use a disease-specific tool is a limitation in this study.

\section{Conclusions}

Our findings provide tentative evidence that physical activity counseling is feasible and appears to increase physical activity and quality of life among children with CF. The intervention was feasible, with good recruitment, retention, adherence, and acceptability rates. The promising results from this feasibility trial support the development of a suitably powered randomized controlled trial in the future. To fully power the study, it will probably need to be multi-sited in nature, offered at all Canadian CF centers. A fully powered efficacy trial of CF Chatters is now needed to examine whether physical activity counseling promotes sustained physical activity participation in what continues to be an extremely challenging illness to treat.

\section{REFERENCES}

1. Stevens D, Oades PJ, Armstrong N, Williams CA. A survey of exercise testing and training in UK cystic fibrosis clinics. J Cyst Fibros 2010;9(5):302-306.

2. van Doorn N. Exercise programs for children with cystic fibrosis: a systematic review of randomized controlled trials. Disabil Rehabil 2010;32(1):41-49.

3. Schneiderman-Walker J, Pollock SL, Corey M, Wilkes DD, Canny GJ, Pedder L, Reisman JJ. A randomized controlled trial of a 3-year home exercise program in cystic fibrosis. J Pediatr 2000;136(3):304310 . 


\section{Physical Activity Counseling in CF}

4. Orenstein DM, Hovell MF, Mulvihill M, Keating KK, Hofstetter CR, Kelsey S, et al. Strength vs aerobic training in children with cystic fibrosis: a randomized controlled trial. Chest 2004;126(4):1204-1214.

5. Moorcroft AJ, Dodd ME, Morris J, Webb AK. Individualized unsupervised exercise training in adults with cystic fibrosis: a 1 year randomized controlled trial. Thorax 2004;59(12):1074-1080.

6. Selvadurai HC, Blimkie CJ, Meyers N, Mellis CM, Cooper PJ, Van Asperen PP. Randomized controlled study of in-hospital exercise training programs in children with cystic fibrosis. Pediatr Pulmonol 2002;33(3):194-200.

7. Klijn PH, Oudshoorn A, van der Ent CK, van der Net J, Kimpen JL, Helders PJ. Effects of anaerobic training in children with cystic fibrosis: a randomized controlled study. Chest 2004;125(4):12991305.

8. Santana Sosa E, Groeneveld IF, Gonzalez-Saiz L, López-Mojares LM, Villa-Asensi JR, Barrio Gonzalez MI, et al. Intrahospital weight and aerobic training in children with cystic fibrosis: a randomized controlled trial. Med Sci Sports Exerc 2012;44(1):2-11.

9. Rovedder PM, Flores J, Ziegler B, Casarotto F, Jaques P, Barreto SS, Dalcin Pde T. Exercise programme in patients with cystic fibrosis: a randomized controlled trial. Respir Med 2014;108(8):1134-1140.

10. Schindel CS, Hommerding PX, Melo DA, Baptista RR, Marostica PJ, Donadio MV. Physical exercise recommendations improve postural changes found in children and adolescents with cystic fibrosis: a randomized controlled trial. J Pediatr 2015;166(3):710-716.e2.

11. Lorenc A, Ronan P, Mian A, Madge S, Carr SB, Agent P, et al. Cystic fibrosis-children and adults Tai Chi study (CF CATS2): can Tai Chi improve symptoms and quality of life for people with cystic fibrosis? second phase study protocol. Chin J Integr Med 2015 [Epub ahead of print]. doi: 10.1007/s11655-015-2150-1.

12. Moorcroft AJ, Dodd ME, Webb AK. Exercise testing and prognosis in adult cystic fibrosis. Thorax 1997;52(3):291-293.

13. Pianosi P, Leblanc J, Almudevar A. Peak oxygen uptake and mortality in children with cystic fibrosis. Thorax 2005;60(1):50-54.

14. Prasad SA, Cerny FJ. Factors that influence adherence to exercise and their effectiveness: application to cystic fibrosis. Pediatr Pulmonol 2002;34(1):66-72.

15. Moola F, Faulkner GE, Schneiderman JE. "CF chatters": the development of a theoretically informed physical activity intervention for youth with cystic fibrosis. OJPM 2011;1(3):109-124. doi: 10.4236/ ojpm.2011.13016.

16. Turchetta A, Salerno T, Lucidi V, Libera F, Cutrera R, Bush A. Usefulness of a program of hospital-supervised physical training in patients with cystic fibrosis. Pediatr Pulmonol 2004;38(2):115-118.

17. Gulmans VA, de Meer K, Brackel HJ, Faber JA, Berger R, Helders PJ. Outpatient exercise training in children with cystic fibrosis: physiological effects, perceived competence, and acceptability. Pediatr Pulmonol 1999;28(1):39-46.

18. Verschuren O, Ketelaar M, Gorter JW, Helders PJ, Uiterwaal CS, Takken T. Exercise training program in children and adolescents with cerebral palsy: a randomized controlled trial. Arch Pediatr Adolesc Med 2007;161(11):1075-1081.

19. Kahn EB, Ramsey LT, Brownson RC, Heath GW, Howze EH, Powell KE, et al. The effectiveness of interventions to increase physical activity: a systematic review. Am J Prev Med 2002;22(4 Suppl):73107.

20. Rhodes RE, Pfaeffli LA. Mediators of physical activity behaviour change among adult non-clinical populations: a review update. Int J Behav Nutr Phys Act 2010;7(1):37.
21. Campbell M, Fitzpatrick R, Haines A, Kinmonth AL, Sandercock P, Spiegelhalter D, Tyrer P. Framework for design and evaluation of complex interventions to improve health. BMJ 2000;321(7262):694696.

22. Loughlan C, Mutrie N. Conducting an exercise consultation: guidelines for health professionals. J Inst Health Educ 1996;33(3):78-82.

23. Wang X, Dockery DW, Wypij D, Fay ME, Ferris BG Jr. Pulmonary function between 6 and 18 years of age. Pediatr Pulmonol 1993; 15(2):75-88.

24. Colley RC, Garriguet D, Janssen I, Craig CL, Clarke J, Tremblay MS. Physical activity of Canadian adults: accelerometer results from the 2007 to 2009 Canadian Health Measures Survey. Health Rep 2011;22(1):7-14

25. Aznar S, Gallardo C, Fiuza-Luces C, Santana-Sosa E, López-Mojares LM, Santalla A, et al. Levels of moderate-vigorous physical activity are low in Spanish children with cystic fibrosis: a comparison with healthy controls. J Cyst Fibros 2014;13(3):335-340.

26. Longmuir PE, Russell JL, Corey M, McCrindle BW. Factors associated with the objectively measured activity levels for children after the Fontan procedure. Circulation 2007;116(Suppl 16):479-480.

27. Longmuir PE, Corey M, Faulkner G, Russell JL, McCrindle BW. Children after fontan have strength and body composition similar to healthy peers and can successfully participate in daily moderate-tovigorous physical activity. Pediatr Cardiol 2015;36(4):759-767.

28. Stone MR, Rowlands AV, Eston RG. Characteristics of the activity pattern in normal weight and overweight boys. Prev Med 2009; 49(2):205-208.

29. Stone MR, Rowlands AV, Middlebrooke AR, Jawis MN, Eston RG. The pattern of physical activity in relation to health outcomes in boys. Int J Pediatr Obes 2009;4(4):306-315.

30. Evenson KR, Catellier DJ, Gill K, Ondrak KS, McMurray RG. Calibration of two objective measures of physical activity for children. J Sports Sci 2008;26(14):1557-1565.

31. Modi AC, Lim CS, Driscoll KA, Piazza-Waggoner C, Quittner AL, Wooldridge J. Changes in pediatric health-related quality of life in cystic fibrosis after IV antibiotic treatment for pulmonary exacerbations. J Clin Psychol Med Settings 2010;17(1):49-55.

32. Leon AC, Davis LL, Kraemer HC. The role and interpretation of pilot studies in clinical research. J Psychiatr Res 2011;45(5):626-629.

33. Thabane L, Ma J, Chu R, Cheng J, Ismaila A, Rios LP, et al. A tutorial on pilot studies: the what, why and how. BMC Med Res Methodol 2010;10:1.

34. van Teijlingen E, Hundley V. The importance of pilot studies. Nurs Stand 2002;16(40):33-36.

35. Saha C, Jones MP. Bias in the last observation carried forward method under informative dropout. J Stat Plan Inference 2009;139(2): 246-255.

36. Matthews CE, Chen KY, Freedson PS, Buchowski MS, Beech BM, Pate RR, Troiano RP. Amount of time spent in sedentary behaviors in the United States, 2003-2004. Am J Epidemiol 2008;167(7):875-881.

37. Moola F, Faulkner GE, Kirsh JA, Kilburn J. Physical activity and sport participation in youth with congenital heart disease: perceptions of children and parents. Adapt Phys Activ Q 2008;25(1):49-70.

38. Corey M, Edwards L, Levison H, Knowles M. Longitudinal analysis of pulmonary function decline in patients with cystic fibrosis. J Pediatr 1997;131(6):809-814

39. Berge J, Patteron J, Goetz D, Milla C. Gender differences in young adults' perceptions of living with cystic fibrosis during the transition to adulthood: a qualitative investigation. Families Syst Health 2007; 25(2):190-203. doi: 10.1037/1091-7527.25.2.190. 